\title{
Frequency-dependent electrical characteristics of DNA using molecular dynamics simulation
}

\author{
Makoto Ikeda ${ }^{1,3}$, Kazuo Nakazato ${ }^{2}$, Hiroshi Mizuta ${ }^{2}$, \\ Martin Green $^{1}$, David Hasko ${ }^{1}$ and Haroon Ahmed ${ }^{1}$ \\ ${ }^{1}$ Microelectronics Research Centre, Cavendish Laboratory, University of Cambridge, \\ Madingley Road, Cambridge CB3 OHE, UK \\ ${ }^{2}$ Hitachi Cambridge Laboratory, Hitachi Europe Ltd., Madingley Road, \\ Cambridge CB3 OHE, UK \\ E-mail: ikeda@silicon.u-tokyo.ac.jp
}

Received 17 September 2002, in final form 2 December 2002

Published 10 January 2003

Online at stacks.iop.org/Nano/14/123

\begin{abstract}
Research on DNA has been widely carried out as a promising material for nanoelectronics, medicine development and disease diagnosis, using experiment, simulation and theory. We have carried out molecular dynamics simulation coupled with the linear response theory, based on the time-correlation function of an observable, in order to extract the frequency-dependent electrical characteristics of DNA. We observe a dielectric relaxation at around $50 \mathrm{MHz}$ in the case of octamers-DNA, which corresponds to a delta-relaxation. We also observe dielectric relaxation in the case of mixtures of DNA, water molecules and ions, given by the superposition of the individual dielectric relaxations of the DNA and the bulk-like water, at frequencies of about $50 \mathrm{MHz}$ and $10 \mathrm{GHz}$, respectively.
\end{abstract}

\section{Introduction}

Research on DNA has been widely carried out as a promising material for nanoelectronics, medicine development and disease diagnosis, using experiment, simulation and theory. With the completion of the human genome project, a new era of opportunity is brought to molecular biology and genetics. New detection methods for DNA are required, in order to enable devices to collect data at high throughput and in real time. For this purpose, several new techniques have been proposed, such as using cantilevers for the detection of steric forces caused by the hybridization of target DNA with probe DNA immobilized onto a surface [1-15]. For label-free detection, it is essential to know the precise electrical characteristics of DNA. Many experimental results and theoretical calculations on the conductivity of DNA have been published, showing a wide variety of behaviour, from insulating to metallic conduction. Despite this, the conductivity of DNA remains a matter for investigation [16-36].

\footnotetext{
3 Address for correspondence: VLSI Design and Education Centre, University of Tokyo, 7-3-1 Hongo, Bunkyo-ku, Tokyo 113-8656, Japan.
}

In this paper, we describe a technique to calculate the electrical characteristics of DNA, which can be applied to label-free DNA detection. We have employed linear response theory in a molecular dynamics simulation, to extract the frequency-dependent electrical characteristics.

In the next section, we describe the linear response theory. In section 3, we describe the molecular dynamics simulation and the simulated systems. In section 4, we describe the results for the frequency-dependent electrical characteristics of DNA.

\section{Linear response theory}

Linear response theory defines a linear relationship between the macroscopic characteristics and the microscopic observables, in quasi-equilibrated and uniform systems [37-40].

For the explanation of dielectric phenomena, linear response theory deals with the response of a macroscopic system to an applied external electric field. On the macroscopic level, linear response theory assumes a linear relationship between the applied external field $\boldsymbol{E}_{0}(\omega)$ and the ensemble average of an observable $\langle\boldsymbol{O}(\omega)\rangle$ :

$$
\langle\boldsymbol{O}(\omega)\rangle=\chi_{O P}(\omega) \boldsymbol{E}_{0}(\omega) .
$$






Figure 1. The simulated time-correlation function $\langle M(0) M(t)\rangle$ of a DNA molecule (crosses) and linear-type (dashed line) and exponential-type (continuous curve) approximation curves

$\chi_{O P}(\omega)$ is the complex generalized susceptibility, which describes the coupling of the observable $\boldsymbol{O}(t)$ to the polarization $\boldsymbol{P}(t)$ of the system. According to linear response theory, the generalized susceptibility for an observable $\boldsymbol{O}(t)$ is defined as

$$
\begin{aligned}
& \chi_{O P}(\omega)=1 / 3 k T\left[\langle\boldsymbol{O}(0) \boldsymbol{M}(0)\rangle-\mathrm{j} \omega\langle\boldsymbol{O}(0) \boldsymbol{M}(t)\rangle_{\omega}\right. \\
& \left.\quad+\langle\boldsymbol{O}(0) \boldsymbol{J}(t)\rangle_{\omega}\right]
\end{aligned}
$$

where $\boldsymbol{M}(t)=\Sigma q_{i} \boldsymbol{r}_{i}(t)$ is the dipole moment due to charges $q_{i}$ and displacement $\boldsymbol{r}_{i}(t)$; and $\boldsymbol{J}(t)=\Sigma q_{i} \boldsymbol{v}_{i}(t)$ is the ion current due to charges $q_{i}$ moving with velocity $\boldsymbol{v}_{i}(t)$. $\langle\boldsymbol{A}(0) \boldsymbol{B}(t)\rangle$ denotes a time-correlation function of the timedependent quantities $\boldsymbol{A}(t)$ and $\boldsymbol{B}(t)$, and $\langle\boldsymbol{A}(0) \boldsymbol{B}(t)\rangle_{\omega}$ denotes a Laplace transform of the time-correlation function. The above equations use a general observable $\boldsymbol{O}(t)$, which can be specified as the polarization of the molecules $\boldsymbol{P}(t)=$ $1 / V M(t)$ or as the current density of the ions $I(t)=$ $1 / V \boldsymbol{J}(t)$, where $V$ is the simulation volume, giving

$$
\langle\boldsymbol{P}(\omega)\rangle=\chi_{P P}(\omega) \boldsymbol{E}_{0}(\omega)
$$

with

$$
\begin{aligned}
& \chi_{P P}(\omega)=1 / 3 V k T\left[\langle\boldsymbol{M}(0) \boldsymbol{M}(0)\rangle-\mathrm{j} \omega\langle\boldsymbol{M}(0) \boldsymbol{M}(t)\rangle_{\omega}\right. \\
& \left.\quad+\langle\boldsymbol{M}(0) \boldsymbol{J}(t)\rangle_{\omega}\right]
\end{aligned}
$$

and

$$
\langle\boldsymbol{I}(\omega)\rangle=\chi_{i P}(\omega) \boldsymbol{E}_{0}(\omega)
$$

with

$$
\chi_{i P}(\omega)=1 / 3 V k T\left[-\mathrm{j} \omega\langle\boldsymbol{J}(0) \boldsymbol{M}(t)\rangle_{\omega}+\langle\boldsymbol{J}(0) \boldsymbol{J}(t)\rangle \omega\right] .
$$

The phenomenological equations of matter, $\boldsymbol{P}(\omega)=(\varepsilon(\omega)-$ 1) $\boldsymbol{E}(\omega) / 4 \pi$ and $\boldsymbol{I}(\omega)=\sigma(\omega) \boldsymbol{E}(\omega)$, define the dielectric constant $\varepsilon(\omega)$ and the conductivity $\sigma(\omega)$. Both involve the Maxwell field $\boldsymbol{E}(\omega)$, which is the electric field acting inside a macroscopic piece of matter. In the case of the ideal Ewald summation, where the cut-off radius $r_{c}$ and decay parameter $\eta$ are both infinite, the Maxwell field is equal to the externally applied electric field $\boldsymbol{E}_{0}(\omega)$, and the dielectric constant and conductivity can be expressed as $\varepsilon(\omega)=1+4 \pi \chi_{P P}(\omega)$ and $\sigma(\omega)=\chi_{i P}(\omega)$. Note that the conductivity $\sigma(\omega)$, in this case, is independent of the dielectric constant, represented by the susceptibility $\chi_{P P}(\omega)$.

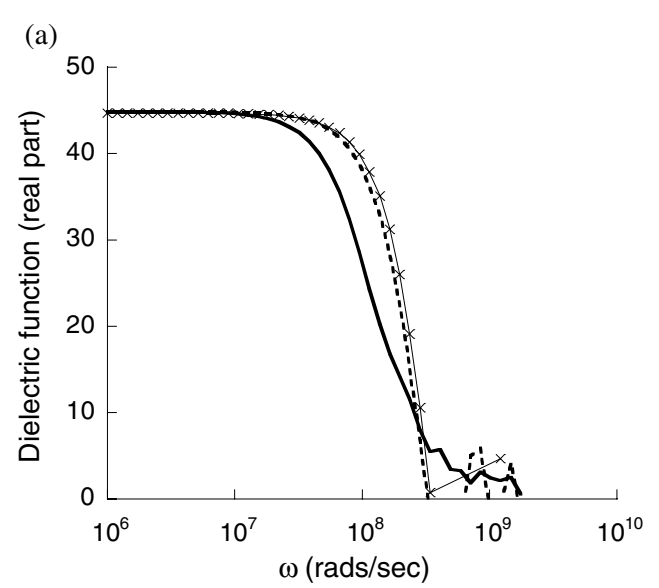

(b)

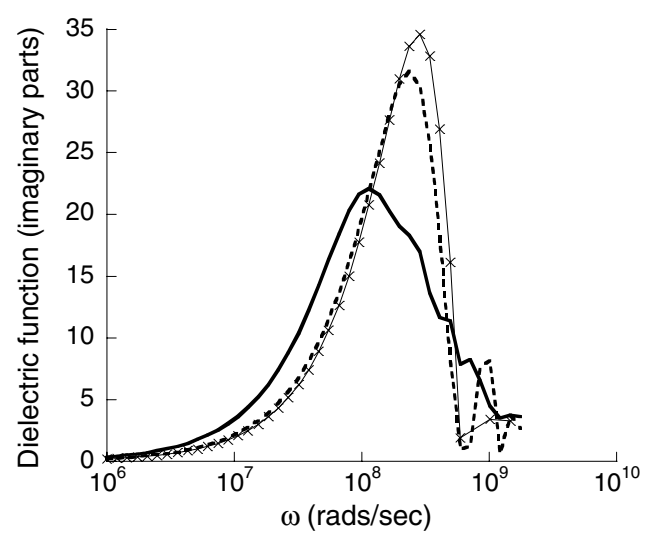

Figure 2. The frequency-dependent dielectric function: (a) real part and (b) imaginary part and linear-type (dashed curve) and exponential-type (continuous curve) approximation curves.

\section{Molecular dynamics simulation}

Molecular dynamics simulation has been widely used for studying molecular structures. In this work, we use TINKER from Washington University, which employs a smooth particle-mesh Ewald summation method.

We used octamer randomly generated ds/ss-DNA d[GTAGCAAA] in this simulation. The simulation system size corresponds to a DNA surface density of 9.6 molecules $\mathrm{nm}^{-2}$, and a few layers of water molecules occupy the gaps between adjacent DNA molecules. Each DNA backbone requires $14 \mathrm{Na}^{+}$counter-ions, and two $\mathrm{Na}^{+}$ions with two $\mathrm{Cl}^{-}$ions corresponding to a $0.1 \mathrm{M}$ saline solution, similar to many DNA buffer solutions. A summary of the simulation is listed in table 1 .

\section{Simulation results}

Figure 1 shows the time-correlation function of a DNA molecule. A linear-type approximation describes the behaviour well, $F_{\text {lin }}(\omega)=C_{0 l}+C_{l 1} \exp \left(\frac{-t}{\tau_{11}}\right)+C_{2 l} t$, as does an exponential-type approximation, $F_{\exp }(\omega)=C_{0 e} \exp \left(\frac{-t}{\tau_{1 e}}\right)+$ $C_{1 e} \exp \left(\frac{-t}{\tau_{2 e}}\right)$, where $C_{0 l}, C_{1 l}, C_{2 l}, \tau_{1 l}, C_{0 e}, C_{1 e}, \tau_{1 e}$ and $\tau_{2 e}$, are respectively $781.4,3.6,-0.08,0.8,781.4,3.6,8000$ and $0.8 \mathrm{ps}$. The frequency-dependent dielectric behaviour is shown in figure 2. Figure 3 shows the time-correlation function 

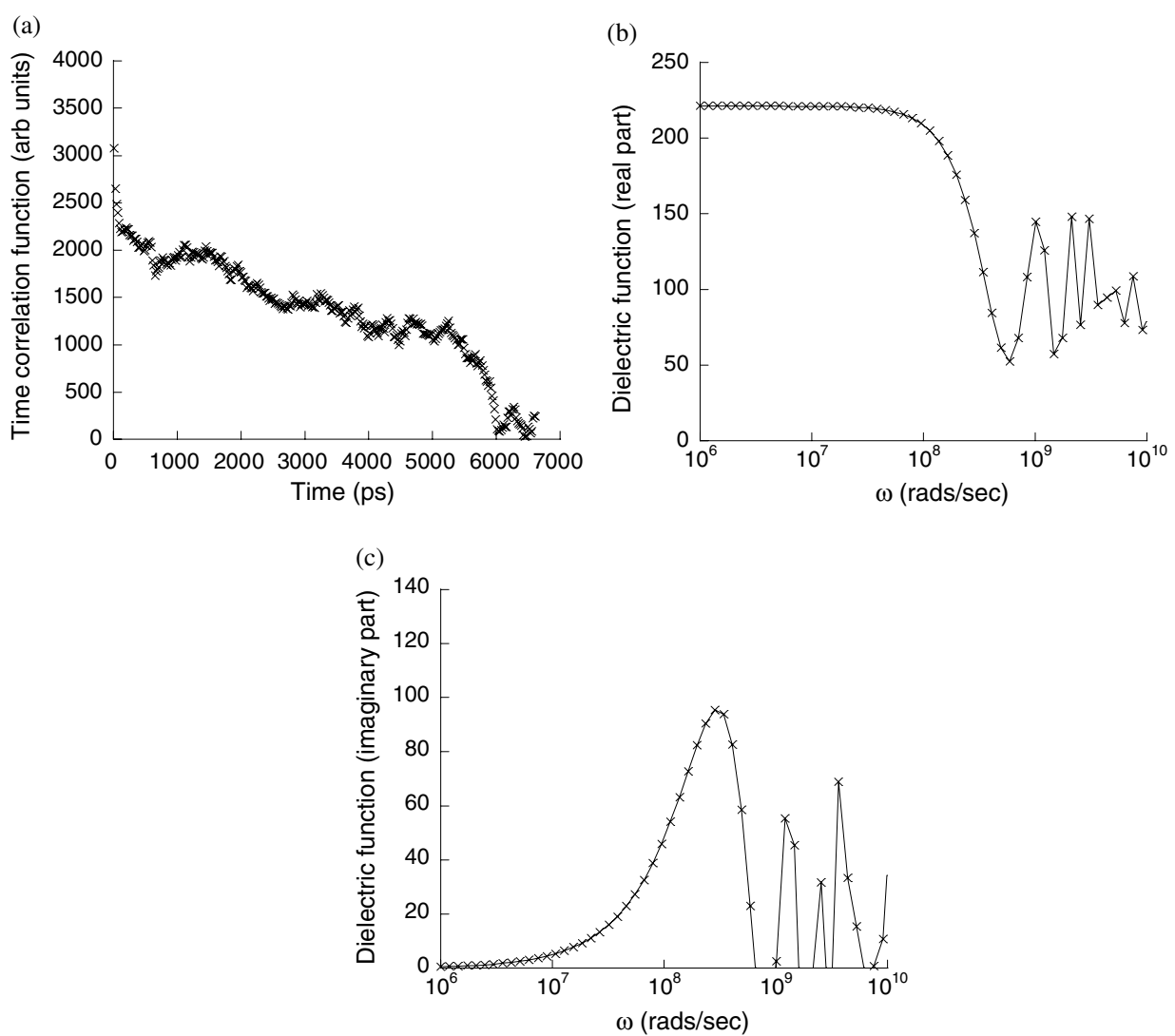

Figure 3. (a) The simulated time-correlation function $\langle M(0) M(t)\rangle$ and (b), (c) the real and imaginary parts of the dielectric function for a mixture of DNA and water molecules.

Table 1. Simulation set-up.

\begin{tabular}{|c|c|}
\hline Force field & $\begin{array}{l}\text { Lennard-Jones } 12-6 \text { potential } \\
\text { (amber } 94 \text { parameter set) }\end{array}$ \\
\hline System size & $3.107 \times 3.107 \times 4.350 \mathrm{~nm}^{3}$ \\
\hline Molecules & $\begin{array}{l}\text { Octamer random sequence ds-DNA, } \\
1239 \text { water molecules, } \\
16 \mathrm{Na}^{+}, 2 \mathrm{Cl}^{-} \text {, total } 4244 \text { atoms }\end{array}$ \\
\hline Temperature & $298 \mathrm{~K}$ \\
\hline Time step & $1 \mathrm{fs}$ \\
\hline Cut-offs & Lennard-Jones, $0.9 \mathrm{~nm}$; Ewald, $0.9 \mathrm{~nm}$ \\
\hline Simulation period & $\begin{array}{l}600 \mathrm{ps} \text { for equilibrium, } 7.3 \mathrm{~ns} \text { for } \\
\text { correlation function }\end{array}$ \\
\hline
\end{tabular}

and the dielectric behaviour for a mixture of DNA and water molecules. Figure 4 shows the time-correlation function and the dielectric behaviour for water molecules only.

Figure 3 shows that the characteristic frequency of the DNA is between 18 and $27 \mathrm{MHz}$, which is consistent with the $\delta$-relaxation expected in the range $1 \mathrm{MHz}-1 \mathrm{GHz}$, due to the orientational change of strongly bound water, or due to short scale relaxations of the DNA chain. On the other hand, the characteristic frequency of water molecules, as seen in figure 4, is nearly the same as that of bulk water, which means that the few layers of water molecules present still demonstrate bulklike characteristics. The characteristic frequency of the molecule mixture seems to be a superposition of those due to DNA and water individually.

It has been reported that there are at least three kinds of relaxation for DNA [41].
(1) $\alpha$-relaxation. Less than a few $\mathrm{kHz}$. A large dielectric increment whose characteristic frequency is dependent on the size of the DNA molecule. Relaxation seems to reflect the migrations of counter-ions along the entire length of the DNA molecule.

(2) $\delta$-relaxation. $1 \mathrm{MHz}-1 \mathrm{GHz}$. A smaller molecular weight independent dielectric increment. This has been attributed to orientational change of strongly bound water, or the short scale relaxations of the DNA chain.

(3) $\gamma$-relaxation. $1 \mathrm{GHz}$ or above. Reorientation of dipolar water molecules.

Recently published DNA measurements using a 35-mer of poly-T and poly-G DNA in nanogap junctions demonstrate similar dielectric decay characteristics to those simulated here [42]. The characteristic frequency from these experimental results differs markedly from the simulated results. Such a difference may be caused by the length and surface density of the DNA used or differences in the geometry, in the extent of the simulated region and time or in the relaxation mechanism.

\section{Conclusions}

In this work, we demonstrate the possibility of extracting the electrical characteristics of DNA using molecular dynamics simulation combined with linear response theory. Results show agreement with previously reported measurements on the $\delta$-relaxation frequency of DNA and water molecules, and the latter show similar characteristics to of bulk water. 
(a)

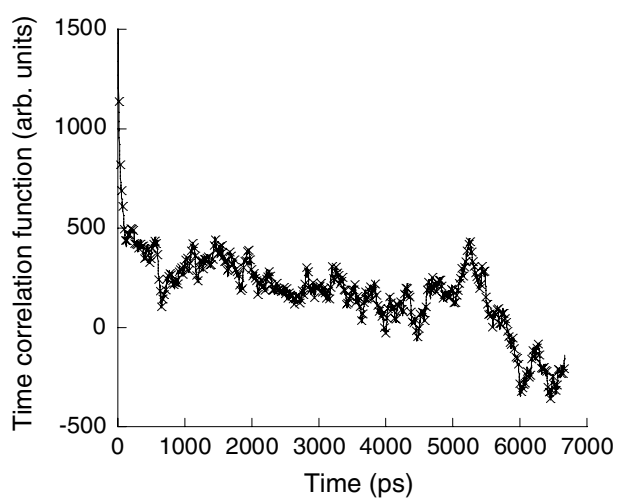

(b)

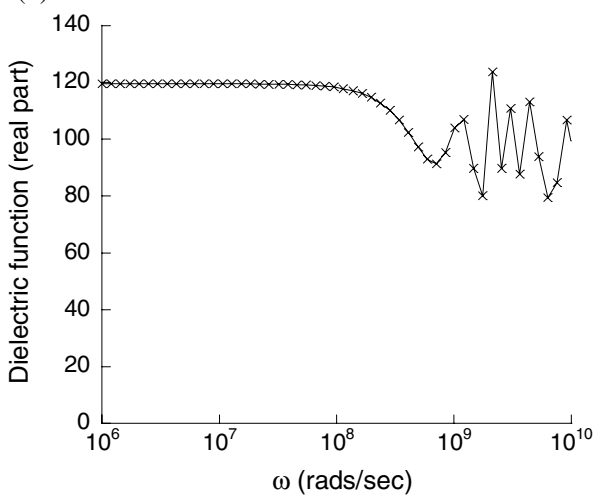

(c)

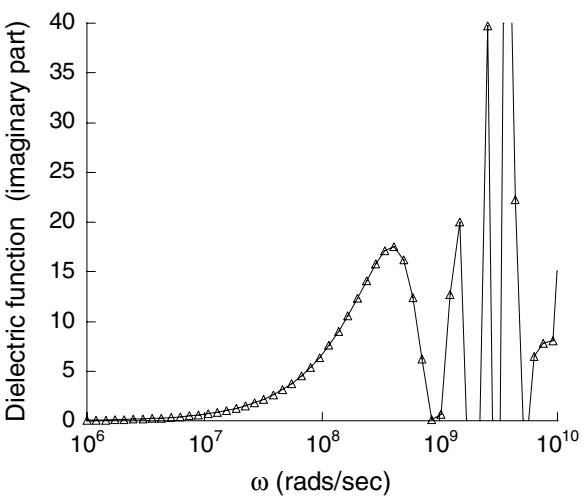

Figure 4. (a) The simulated time-correlation function $\langle M(0) M(t)\rangle$ and (b), (c) the real and imaginary parts of the dielectric function for water molecules only.

The simulation system in this work is not large enough to obtain precise characteristics; however, some agreement with experimental results is obtained.

\section{References}

[1] Ihara T, Maruo Y, Takenaka S and Takagi M 1996 Nucl. Acids Res. 244273

[2] Arlinghaus H F and Kwoka M N 1997 Anal. Chem. 693747

[3] Sosnowski R G, Tu E, Butler W F, O'Connell J P and Heller M J 1997 Proc. Natl Acad. Sci. USA 941119

[4] Yang M, McGovern M E and Thompson M 1997 Anal. Chim. Acta $\mathbf{3 4 6} 259$

[5] O'Donnell M J, Tang K, Koster H, Smith C L and Cantor C R 1997 Anal. Chem. 692438

[6] Lang H P, Berger R, Andreoli C, Brugger J, Despont M, Vettiger P, Gerber Ch, Gimzewski J K, Ramseyer J P, Meyer E and Guntherodt H J 1998 Appl. Phys. Lett. 72383

[7] Cheng J, Sheldon E L, Wu L, Uribe A, Gerrue L O, Carrino J, Heller M J and O'Connell J P 1998 Nat. Biotechnol. 16541

[8] Steel A B, Herme T M and Tarlov M J 1998 Anal. Chem. 70 4670

[9] Shlyakhtenko L S, Gall A A, Weimer J J, Hawn D D and Lybchenko Y L 1999 Biophys. J. 77568

[10] Schmalzing D, Koutny L, Salas-Solano O, Adourian A Matsudaira P and Ehrlich D 1999 Electrophoresis 203066

[11] Berggren C, Stalhandske P, Brundell J and Johansson G 1999 Electroanalysis 11156

[12] Takenaka S, Yamashita K, Takagi M, Uto Y and Kondo H 2000 Anal. Chem. 721334

[13] Fritz J, Baller M K, Lang H P, Rothuizen H, Vettiger P, Mayer E, Guntherodt H J, Gerber Ch and Gimzewski J K 2000 Science $\mathbf{2 8 8} 316$
[14] Salas-Solano O, Schmalzing D, Koutny L, Buonocore S, Adourian A, Matsudaira P and Ehrlich D 2000 Anal. Chem. 723129

[15] Koutny L, Schmalzing D, Salas-Solano O, El-Difrawy S Adourian A, Buonocore S, Abbey K, McEwan P and Ehrlich D 2000 Anal. Chem. 723388

[16] Lindsay S M, Li Y, Pan J, Thundat T, Nagahara L A, Oden P, DeRose J A and Knipping U 1991 J. Vac. Sci. Technol. B 9 1096

[17] Murphy C J, Arkin M R, Jenkins Y, Ghatlia N D, Bossmann S H, Turro N J and Barton J K 1993 Science 2621025

[18] Warman J M, de Haas M P and Rupprecht A 1996 Chem. Phys. Lett. 249319

[19] Armitage B, Ly D, Koch T, Frydenlund H, Orum H, Batz H G and Schuster G B 1997 Proc. Natl Acad. Sci. USA 9412320

[20] Meggers E, Michel-Beyerle M E and Giese B 1998 J. Am. Chem. Soc. $\mathbf{1 2 0} 12950$

[21] Jortner J, Bixon M, Langenbacher T and Michl-Beyerle M E 1998 Proc. Natl Acad. Sci. USA 9512759

[22] Fiebig T, Wan C, Kelley S O, Barton J K and Zewail A H 1999 Proc. Natl Acad. Sci. USA 961187

[23] Giese B, Wessely S, Spormann M, Lindemann U, Meggers E and Michel-Beyerle M E 1999 Angew. Chem., Int. Edn. Engl. 38996

[24] Kelley S O, Jackson N M, Hill M G and Barton J K 1999 Angew. Chem., Int. Edn. Engl. 38941

[25] Henderson P T, Jones D, Hampikian G, Kan Y and Schuster G B 1999 Proc. Natl Acad. Sci. USA 968353

[26] Harriman A 1999 Angew. Chem., Int. Edn. Engl. 38945

[27] Messer A, Carpenter K, Forzley K, Buchanan J, Yang S, Razskazovskii Y, Cai Z and Sevilla M D 2000 J. Phys. Chem. B 1041128

[28] Tavernier H L and Fayer M D 2000 J. Phys. Chem. 10411541

[29] Voityuk A A, Rosch N, Bixon M and Jortner J 2000 J. Phys. Chem. 1049740 
[30] Segal D, Nitzan A, Davis W B, Wasielewski M R and Ratner M A 2000 J. Phys. Chem. 1043817

[31] de Pablo P J, Colchero J, Gomez-Herrero J, Martinez M T and Baro A M 2000 TNT2000

[32] Porath D, Bezryadin A, de Vries S and Dekker C 2000 Nature 403635

[33] Fink H-W and Schonenberger C 1999 Nature 398407

[34] Grozema F C, Berlin Y A and Siebbeles L D A $2000 \mathrm{~J}$. Am. Chem. Soc. 12210903

[35] Berlin Y A, Burin A L and Ratner M A 2000 Superlatt. Microstruct. 28241
[36] Wan C, Fiebig T, Schiemann O, Barton J K and Zewail A H 2000 Proc. Natl Acad. Sci. USA 9714052

[37] Allnatt A R and Allnatt E L 1991 Phil. Mag. Lett. 64393

[38] Chandra A, Wei D and Patey G N 1993 J. Chem. Phys. 98 4959

[39] Chandra A and Bagchi B 2000 J. Chem. Phys. 1121876

[40] Chandra A and Bagchi B 2000 J. Phys. Chem. B 1049067

[41] Saif B, Mohr R K, Montrose C J and Litovitz T A 1991 Biopolymers $\mathbf{3 1} 1171$

[42] Lee J, Oh S, Choi Y-K and Lee L 2002 Micro Total Analysis Systems ( $\mu$ TAS2002) I (Dordrecht: Kluwer) p 305 